\title{
Altered functional connectivity in patients with post-stroke memory impairment: A resting fMRI study
}

\author{
JIAO LIU ${ }^{1,2^{*}}$, QIN WANG ${ }^{1 *}$, FEIWEN LIU ${ }^{1}$, HAIYAN SONG $^{1}$, XIAOFENG LIANG $^{1}$, ZHENGKUN LIN $^{1}$,

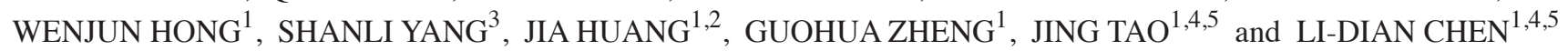 \\ ${ }^{1}$ College of Rehabilitation Medicine, Fujian University of Traditional Chinese Medicine; \\ ${ }^{2}$ Fujian Rehabilitation Tech Co-innovation Center, Fuzhou, Fujian 350122; ${ }^{3}$ Fujian University of Traditional Chinese Medicine \\ Subsidiary Rehabilitation Hospital; ${ }^{4}$ Key Laboratory of Motor Rehabilitation of Fujian, Fuzhou, Fujian 350003; \\ ${ }^{5}$ Fujian Provincial Rehabilitation Industrial Institution, Fuzhou, Fujian 350000, P.R. China
}

Received February 14, 2016; Accepted February 17, 2017

DOI: $10.3892 / \mathrm{etm} .2017 .4751$

\begin{abstract}
Post-stroke memory dysfunction (PMD) is one of the most common forms of cognitive impairment among stroke survivors. However, only a limited number of studies have directly investigated the neural mechanisms associated with memory decline. The aim of the present study was to identify dynamic changes in the functional organization of the default mode network (DMN) and the dorsal attention network of patients with PMD. A total of 27 patients with PMD who experienced a stroke in the right hemisphere were enrolled in the current study, along with 27 healthy control subjects matched by age, sex, and educational level. A behavioral examination and functional magnetic resonance imaging scan were performed. The data were analyzed using an independent component analysis method. The results revealed a significantly increased functional connectivity between the DMN and prefrontal cortex (left middle/inferior frontal and left precentral gyri), temporal regions (left superior temporal gyrus), and bilateral and posterior cingulate gyri/precuneus $(\mathrm{P}<0.001)$. There was also a significantly decreased functional connectivity between the DMN and right middle temporal gyrus, left uvula, and right inferior parietal lobule, and between the dorsal attention network and prefrontal cortex (left precentral/inferior and right inferior/middle frontal gyri), right inferior parietal gyrus, and right insula $(\mathrm{P}<0.001)$. These results suggest that the stroke affected both the lesioned and contralesional hemispheres. The prefrontal cortex, temporal
\end{abstract}

Correspondence to: Dr Jing Tao or Dr Li-Dian Chen, College of Rehabilitation Medicine, Fujian University of Traditional Chinese Medicine, 1 Huatuo Road, Fuzhou, Fujian 350122, P.R. China

E-mail: taojing01@163.com

E-mail: fjtcm1958@sina.com

${ }^{*}$ Contributed equally

Key words: memory disorders, functional magnetic resonance imaging, functional connectivity, stroke, independent component analysis regions, insula, and posterior cingulate gyrus/precuneus serve a crucial role in memory processing.

\section{Introduction}

Each year $>15$ million people suffer from stroke, and almost two-thirds of stroke patients exhibit cognitive decline in different domains, including attention, memory and executive function $(1,2)$. Post-stroke memory dysfunction (PMD) is one of the most prevalent cognitive impairments among survivors of stroke, as reported previously (3). PMD greatly influences the restoration of patient motor function and activities of daily living as patients with PMD are unable to fully comprehend complex rehabilitation training instructions and perform the instructions later (4). Rasquin et al (5) and Hochstenbach et al (6) have reported that cognitive impairment after stroke (including subtle deficits) are one of the main causes of disability in daily activities. Tatemichi et al (7) reported that cognitive impairmentis a significant independent risk factor for dependent living after stroke. Furthermore, PMD may have a potentially devastating economic and social impact on patients, ultimately leading to a decreased quality of life and increased likelihood of mortality (8).

Although mechanisms leading to general cognitive impairment have previously been reported (9-11), a limited number of studies have directly investigated the neural mechanisms associated with memory decline. Tuladhar et al (12) found that following a stroke, patients presented with delayed memory dysfunction, decreased functional connectivity in the left medial temporal lobe, posterior cingulate and medial prefrontal cortical areas within the DMN compared with controls. However, this study only used one scale to test delayed memory and the mechanism of PMD remains unclear. Considering the high frequency of memory dysfunction, patient complaints and the potentially negative impact of cognitive impairment on the patient and society, the development of viable and effective therapeutic methods to treat PMD is of great importance. To facilitate accurate and timely diagnoses, prognoses and more effective treatments, identifying the underlying mechanism(s) associated with the development of PMD is of paramount significance. 
In addition to the location of PMD-related brain damage, post-stroke cognitive impairment may be attributable to brain regions far removed from the lesion (9). A possible reason for these remote effects may be the disruption of brain networks that occurs following stroke $(13,14)$. Recently, the importance of the segregation and integration functions of the brain has been identified (15). Dacosta-Aguayo et al (9) found that, compared with healthy control, patients following a stroke have greater DMN activity in the left precuneus and the left anterior cingulate gyrus and also present with a marked impairment in the functional connectivity between the DMN nodes, including the left superior frontal gyrus and posterior cingulate cortex. Following a stroke, individuals also demonstrate positive correlations with the mini mental state examination (MMSE) scores, which is a scale to test the general cognitive function. Lawrence et al (10) also indicated that whiter matter lesions are associated with the cognitive function, including executive function and processing speed in cerebral small vessel disease. Ding et al (11) demonstrated that the functional connectivity of posterior cingulate cortex/precuneus is associated with cognitive function in stroke patients. In order to understand the mechanism(s) associated with PMD, both structural damage to the brain and changes in its functional connectivity must be considered. Neuroimaging studies have revealed a set of dynamically interrelated brain networks that are considered to serve vital roles in cognition such as dorsal attention network (DAN) (16) and memory such as default mode network (DMN) $(12,17)$. These two brain networks are two diametrically opposed; the DMN consists of regions that typically exhibit higher levels of activity during the resting state, whereas activity in the DAN is higher during task-related activity (18). However, there is no unified conclusion about how PMD affects these two brain networks.

In the present study, the DMN and DAN in patients with PMD were examined using independent component analysis (ICA). In particular, the association between network functional connectivity and different brain regions in patients with PMD was examined to clarify the neuropathological mechanism(s) associated with PMD. It was also investigated whether changes in brain network functional connectivity are correlated with the severity of PMD.

\section{Materials and methods}

Participants. The present study was approved by the Ethics Committee of the Fujian University of Traditional Chinese Medicine Subsidiary Rehabilitation Hospital (Fuzhou, China; approval no. 2013KY-005-01) and each participant gave written informed consent, in accordance with the provisions of the Declaration of Helsinki. A total of 29 participants with PMD were enrolled in Fujian University of Traditional Chinese Medicine Subsidiary Rehabilitation Hospital (Fujian, China) from July 2014 to July 2015; All patients were admitted due to suffering their first stroke and the incidence areas were on the right hemisphere, (see Fig. 1 for lesion overlap map) and each patient was investigated at a different time-point, all within 12 months of stroke. Stroke was confirmed in each patient by computed tomography or magnetic resonance imaging (MRI). The MMSE, including 30-point questionnaire items is used as a standard screening tool for cognitive impairment. The present study performed this as previously described (19) and patients with PMD scored $\leq 24$, which means the patient may suffer from some degree of cognitive impairment. Additionally, all patients had memory complaints. Exclusion criteria were as follows: Contraindications in MRI, presence of another disease that may possibly lead to cognitive impairment, history of severe auditory, visual, or language impairment that could affect cognitive examination and a Beck Depression Scale score $>13$ (20). Beck Depression Scale is used to assess the severity of depressive symptoms, including the feeling of being punished, have suicidal thoughts, or decreased interest in sex, over two weeks. The scale includes 21 items, with a total score of 63 points. The total score of 0 to 13 determines that there is no depression (21). A total of 29 healthy controls with no memory complaints, recruited from Fujian University of Traditional Chinese Medicine Subsidiary Rehabilitation Hospital (Fuzhou, China) from July 2014 to July 2015, were matched to patients with PMD by age, sex and education level. Control subjects were excluded if they had a current neurological or psychiatric disease and/or a MMSE score $>24$. All subjects enrolled in this study were right-handed. None of the healthy subjects had a history of neurological or psychiatric disease nor were they taking regular drugs associated with cognitive function.

Functional MRI (fMRI) data were examined to avoid excessive movement or inclusion of MRI artifacts prior to analysis. A total of 2 patients with PMD and 1 healthy subject were excluded due to excessive motion during resting-state fMRI scanning, and 1 healthy subject was excluded due to an absence of resting-state data. Thus, a total of 27 patients with PMD and 27 control subjects were included in the present study. Demographic and clinical characteristics for the included healthy control and PMD groups are presented in Table I. Both groups were well matched in age, education level and sex, with no significant differences between groups.

Neuropsychological data. All subjects underwent MMSE (22) and Wechsler Memory Scale-Chinese revision (WMS-CR) testing (23). The MMSE was performed to assess general cognitive function and the WMS-CR was used to measure memory function. The WMS-CR consisted of three sub functions and 10 subtests as follows: Immediate memory (digit span), short-term memory (including addition, picture, recognition, visual reproduction, associative, learning, touch and comprehension) and long-term memory (forwards and backwards recital of digits 1-100 and addition). Clinical severity of patients with PMD was determined according to the National Institutes of Health Stroke Scale (24). The modified Barthel index was used to measure activity of daily living (25).

SPSS version 18.0 software (SPSS, Inc., Chicago, IL, USA) was used for neuropsychological data analysis. The Shapiro-Wilk test was performed to examine the normality of demographic variables. Continuous variables analysis between PMD and control groups was performed using a t-test for independent and nonparametric independent samples. $\mathrm{P}<0.05$ was considered to indicate a statistically significant difference.

fMRI data acquisition. Images were obtained using a 3.0 Tesla General Electric scanner (GE Healthcare Life Sciences, Chalfont, UK) with an eight-channel phased array head 
Table I. Demographic and clinical characteristics for PMD and healthy control groups.

\begin{tabular}{|c|c|c|}
\hline Characteristics & $\mathrm{HC}(\mathrm{n}=27)$ & $\operatorname{PMD}(\mathrm{n}=27)$ \\
\hline Age, medians (percentile 25-75), years ${ }^{\mathrm{a}}$ & $59(54-62)$ & $57(47-57)$ \\
\hline Educational level, mean $\pm \mathrm{SD}$, years ${ }^{\mathrm{b}}$ & $8.11(4.74)$ & $9.04(3.65)$ \\
\hline \multicolumn{3}{|l|}{ Sex, No. } \\
\hline Male $(\%)$ & $20(74)$ & $20(74)$ \\
\hline NIHSS, mean \pm SD & NA & $7.96 \pm 3.66$ \\
\hline $\mathrm{MBI}$, mean $\pm \mathrm{SD}$ & NA & $51.22 \pm 25.52$ \\
\hline Lesion volume, medians (percentile $25-75$ ), $\mathrm{cm}^{3}$ & NA & $17.17(8.15-25.99)$ \\
\hline \multicolumn{3}{|l|}{ Stroke type } \\
\hline $\mathrm{ICH}$ & NA & 12 \\
\hline Infarction & NA & 15 \\
\hline \multicolumn{3}{|l|}{ Anatomical regions affected } \\
\hline Basal Ganglia & NA & 19 \\
\hline Corona Radiata & NA & 9 \\
\hline Occipital lobes & NA & 4 \\
\hline Temporal lobes & NA & 9 \\
\hline Parietal lobes & NA & 9 \\
\hline Frontal lobes & NA & 9 \\
\hline Thalamus & NA & 5 \\
\hline
\end{tabular}

${ }^{\mathrm{a}} \mathrm{t}=-0.451$ and $\mathrm{P}=0.652 ;{ }^{\mathrm{b}} \mathrm{z}=0.804$ and $\mathrm{P}=0.425$; NIHSS, national institute of health stroke scale; SD, standard deviation; PMD, post-stroke memory dysfunction; HC, healthy control; NA, not applicable; MBI, the Modified Barthel Index; ICH, intracerebral hemorrhage.

Table II. Neuropsychological tests scores for PMD and HC groups.

\begin{tabular}{lcc}
\hline Variable & HC $(\mathrm{n}=27)$ & PMD $(\mathrm{n}=27)$ \\
\hline General cognitive function & & $21(18-22)$ \\
MMSE & $27(25-29)$ & $47(32-60)$ \\
Total WMS score & $88(25-29)$ & $4(2.27)$ \\
Immediate memory & & \\
Digit span & $7.93(2.21)$ & $6(5-8)$ \\
Short-term memory & & $2(0-5)$ \\
Picture & $9(7-10)$ & $2(0-4)$ \\
Recognition & $9(7-12)$ & $5(1-7)$ \\
Visual reproduction & $9(7-12)$ & $6(6-6)$ \\
Associative learning & $7(6-9)$ & $4.70(2.25)$ \\
Touch memory & $9(7-10)$ & $<01^{\mathrm{a}}$ \\
Comprehension memory & $8.70(2.05)$ & $3(0-6)$ \\
Long-term memory & & $4.46(3.65)$ \\
Forward recite digit $(1-100)$ & $9(6-10)$ & $7(2-9)$ \\
Backward recite digit $(100-1)$ & $10.07(3.54)$ & $<.011^{\mathrm{a}}$ \\
Calculation & $11(10-12)$ & $<0.01^{\mathrm{a}}$ \\
\hline
\end{tabular}

All values are the means (standard deviation) or medians (percentile $25-75$ ). $\mathrm{n}=27$ for each group. ${ }^{\mathrm{a}} \mathrm{P}<0.05$. MD, post-stroke memory dysfunction; HC, healthy control; MMSE, mini mental state examination.

coil. The resting-state scan was performed using an echo planar imaging sequence (time resolution=2,100 $\mathrm{msec}$, echo time $=30 \mathrm{msec}$, flip angle $=90^{\circ}$, slice thickness $=3 \mathrm{~mm}$ with a 0.6-mm gap, 42 slices, 64x64 matrix, field of view=200 mm). Subjects were instructed to lie still with their eyes closed without falling asleep, while staying relaxed and not 
thinking of anything in particular. T1 three-dimensional magnetization-prepared rapid gradient-echo imaging was also performed in the same session (echo time $=1.764 \mathrm{msec}$, flip angle $=15^{\circ}$, inversion time $=450 \mathrm{msec}$, slice thickness $=1 \mathrm{~mm}$, field of view $=240 \mathrm{~mm}$ and 164 slices per acquisition). Both behavioral examinations and fMRI scanning were completed within 1 week of enrollment.

fMRI preprocessing. fMRI data preprocessing and analysis were performed using the Oxford Centre for Functional MRI of the Brain's (FMRIB) Software Library (http://www.fmrib .ox.ac.uk/fsl), AFNI (http://afni.nimh.nih.gov/) and Freesurfer (http://surfer.nmr.mgh.harvard.edu/). The first six volumes of every functional time series were removed to allow for stability of the initial MRI signal and to allow participants to acclimate to the circumstances, leaving a total of 174 volumes for further analysis. Non-brain structures were removed and head motion was corrected using the Brain Extraction Tool and the Linear Image Regression Tool in FMRIB, respectively, and a mean functional image was created for each subject. Images were then spatially smoothed using an 8 -mm full width at half maximum Gaussian smoothing kernel in FMRIB. Data were then band-pass filtered from 0.01 to $0.08 \mathrm{~Hz}$ to reduce the influence of low frequency noise (including slow scanner drifts) and effects of higher frequencies, such as respiratory and cardiac signals (26). In addition, each participant's functional data was coregistered to their skull-stripped anatomical image and subsequently segmented. Nine nuisance covariates (global, white matter, and cerebral spinal fluid signals and six head motion parameters) were removed.

fMRI analysis. fMRI data analyses were performed using multivariate exploratory linear optimized decomposition into independent components (MELODIC), which is analyzed based on the FSL Software Library (FSL version 5.0.1; www.fmrib .ox.ac.uk/fsl) to identify functional connectivity. Probabilistic ICA was applied to derive each group's resting-state network activity at 20 components ( $\mathrm{n}=54$ per group). The DMN and DAN were identified according to functional networks identified in previous studies $(12,27)$ and the similarity between the data from the present study and the template networks derived from 1,414 participants was investigated (template networks are available: http://fcon_1000.projects.nitrc.org/).

A dual-regression technique was also used as previously described (27). Firstly, the two priori-defined networks were used as spatial regressors in a general linear model (GLM) to extract each subject's temporal dynamics. These time courses were then used as a set of temporal regressors in the GLM to generate subject-specific maps associated with the different group-level independent components. Finally, group analysis was performed using the subject-specific maps from the second GLM. The results represent the strength of functional connectivity for each voxel within the DMN and DAN.

Additionally, to investigate the association between changes in functional connectivity and cognitive behavior, a regression analysis was performed between the subject-specific network maps and each subject's MMSE and WMS-CR score, separately. Regression analysis was performed using the Oxford Centre for Functional MRI of the Brain's (FMRIB) Software Library (http://www.fmrib.ox.ac.uk/fsl). The current study performed regression analysis (28) using the network connectivity change maps between patients and healthy control subjects and corresponding changes in clinical outcomes. A threshold of voxel-wise $Z>2.3$ and a cluster correction significance threshold of $\mathrm{P}<0.05$ was used. Furthermore, for both analyses, age, sex, and education level were not considered covariates of interest in this GLM.

\section{Results}

Neuropsychological results. Patients with PMD had a statistically significant impairment in general cognitive function as measured by the MMSE $(\mathrm{P}<0.01)$, as well as in memory function measured by the WMS-CR $(\mathrm{P}<0.01)$ compared with healthy controls (Table II).

Between-group analysis of DMN and DAN functional connectivity. Demographic and neuropsychological data were analyzed using SPSS (Tables I and II). The DMN was obtained from ICA data in conformity with earlier studies (27) and included the medial prefrontal, anterior cingulate, bilateral parietal, and precuneus (Pcu)/posterior cingulate cortices (PCC; Fig. 2A). The DAN in the present study was consistent with that of a previous study (29), consisting bilaterally of the intraparietal, precentral, and superior frontal sulci and ventral precentral gyri and middle frontal gyrus (Fig. 2B).

A comparison between PMD and control subjects (stroke $>$ control) revealed significant differences in functional connectivity between the DMN and the following regions $(\mathrm{P}<0.001)$ : The bilateral $\mathrm{PCC} / \mathrm{Pcu}$ and bilateral cingulate, left precentral, left inferior/middle frontal and left superior temporal gyri (Fig. 3; Table III). A comparison of HCs with PMD subjects (control>stroke) found significant functional connectivity differences between the DMN and right middle temporal gyrus, left uvula, and right inferior parietal lobule. Furthermore, no significant functional connectivity differences were observed between the DAN and cortical areas between the PMD and control groups, whereas functional connectivity between the DAN and left precentral, left inferior frontal, right inferior/middle frontal, and right inferior parietal gyri and right insula was significantly higher in the control group as compared with the PMD group (Fig. 4; Table III).

Correlations between functional connectivity and WMS-CR scores. Regression analysis of the DMN and WMS-CR scores revealed a positive association between the DMN and right medial frontal gyri, and left anterior cingulate and a negative association between the DMN and left claustrum and the bilateral cingulate, left inferior frontal and left precentral gyri (Fig. 3; Table IV). Fig. 3 also depicts the correlation between brain region (cingulate gyrus) FC alterations and WMS score changes. That is, Z-Score (functional connectivity alterations between DMN and cingulate gyrus) was negatively associated with the corresponding WMS score changes; the higher the Z-Score, the lower WMS score. It demonstrated that the function of the connection change has a linear correlation with the behavior changes. Regression analysis between the DAN and WMS-CR identified a positive association between the DAN and right precentral, right middle frontal, and right inferior frontal gyri and right insula (Fig. 4; Table IV). Fig. 4 depicts the 


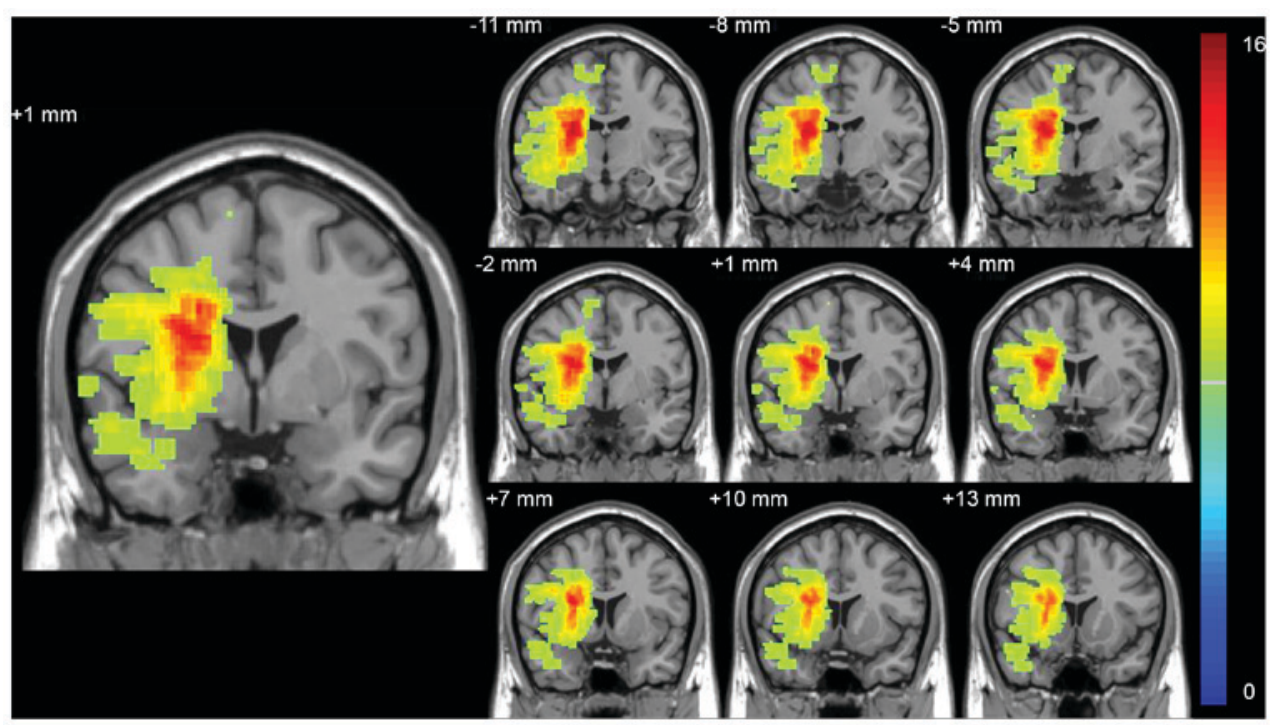

Figure 1. Distribution of the lesion areas for all patients with post-stroke memory dysfunction. The intensity scale refers to the maximum no. of patients with lesions at a particular voxel. The different colors [blue (low) to red (high)] represent the different no. of patients with lesions at a particular voxel and the intensity scale refers to the maximum number of patients with lesions at a particular voxel (the increase no. of patients from blue to red).

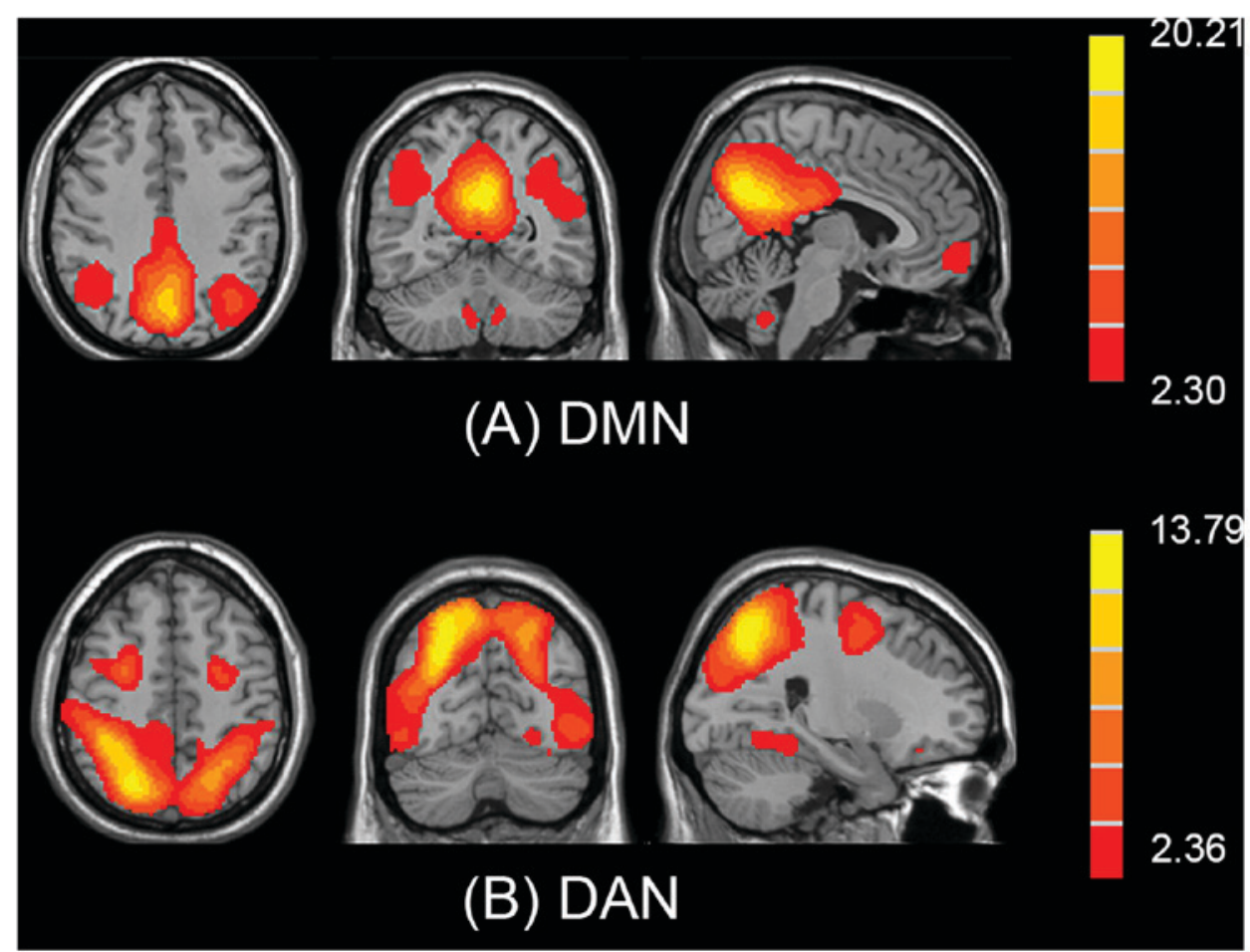

Figure 2. The Z-score maps (red-yellow scale indicates the $\mathrm{z}$ value, increasing from red to yellow), indicate the DMN and DAN, derived from an independent component analysis. (A) DMN derived from independent component analysis. Brain regions observed in the network consist of the medial prefrontal, anterior cingulate, bilateral parietal and precuneus/posterior cingulate cortices. (B) DAN derived from independent component analysis. Brain regions observed in the network covered bilaterally of the intraparietal, precentral, and superior frontal sulci, ventral precentral gyrus, and middle frontal gyri. DMN, default mode network; DAN, dorsal attention network.

correlation between brain region (right inferior frontal gyrus) FC alterations and WMS score changes. Demonstrating that the Z-Score (functional connectivity alterations between DAN and prefrontal) was associated with the corresponding WMS score changes; the higher the Z-Score, the higher WMS score. It demonstrated that the functional connectivity change has a linear correlation with the behavior changes to some extent.
No negative significant associations were observed between the DAN and WMS-CR.

\section{Discussion}

In the present study, ICA methods were used to improve understanding regarding the underlying pathophysiology 
Table III. Brain regions demonstrated significant FC differences between PMD and HC groups.

\begin{tabular}{|c|c|c|c|c|c|c|}
\hline \multirow[b]{2}{*}{ Brain region } & \multirow[b]{2}{*}{ Cluster size } & \multicolumn{3}{|c|}{ MNI coordinates } & \multirow[b]{2}{*}{ Peak Z-Score } & \multirow[b]{2}{*}{ P-value } \\
\hline & & $\mathrm{X}$ & Y & $\mathrm{Z}$ & & \\
\hline \multicolumn{7}{|c|}{ FC between the DMN and brain regions } \\
\hline \multicolumn{7}{|l|}{ Stroke >control } \\
\hline R. cingulate gyrus & 3,355 & 14 & -36 & 34 & 4.3 & $<0.001$ \\
\hline R. precentral gyrus & 1,616 & -50 & -6 & 30 & 4.12 & $<0.001$ \\
\hline L. mid frontal gyrus & 1,202 & -18 & 20 & 66 & 4.04 & $<0.001$ \\
\hline \multicolumn{7}{|l|}{ Control >stroke } \\
\hline R. mid temporal gyrus & 1,878 & 60 & -4 & -16 & 5.01 & $<0.001$ \\
\hline L. uvula & 1,168 & -10 & -90 & -38 & 4.57 & $<0.001$ \\
\hline R. inferior parietal lobule & 1,159 & 46 & -66 & 50 & 4.32 & $<0.001$ \\
\hline \multicolumn{7}{|c|}{ FC between the DAN and brain regions } \\
\hline \multicolumn{7}{|c|}{ Stroke $>$ control } \\
\hline \multicolumn{7}{|l|}{ No result } \\
\hline \multicolumn{7}{|l|}{ Control >stroke } \\
\hline L. precentral gyrus & 4,406 & -48 & 0 & 24 & 4.5 & $<0.001$ \\
\hline R. inferior frontal gyrus & 1,747 & 52 & 6 & 28 & 5.08 & $<0.001$ \\
\hline R. inferior parietal gyrus & 1,280 & 54 & -28 & 34 & 4.05 & $<0.001$ \\
\hline
\end{tabular}

FC, functional connectivity; PMD, post-stroke memory dysfunction; HC, healthy control; MNI, montreal neurological institute; DMN, default mode network; R, right; L, left; DAN, dorsal attention network.

Table IV. Brain regions demonstrated significant associations between WMS and FC.

\begin{tabular}{|c|c|c|c|c|c|c|}
\hline \multirow[b]{2}{*}{ Brain region } & \multirow[b]{2}{*}{ Cluster size } & \multicolumn{3}{|c|}{ MNI coordinates } & \multirow[b]{2}{*}{ Peak Z-Score } & \multirow[b]{2}{*}{ P-value } \\
\hline & & $\mathrm{X}$ & $\mathrm{Y}$ & $\mathrm{Z}$ & & \\
\hline \multicolumn{7}{|c|}{ The FC between the DMN and WMS } \\
\hline \multicolumn{7}{|c|}{ Positive WMS } \\
\hline R. med frontal gyrus & 1,475 & 6 & 52 & -24 & 4.14 & $<0.001$ \\
\hline \multicolumn{7}{|l|}{ Negative WMS } \\
\hline R. cingulate gyrus & 3,705 & 14 & -36 & 36 & 4.7 & $<0.001$ \\
\hline L. claustrum & 3,552 & -30 & 6 & 10 & 4.31 & $<0.001$ \\
\hline L. Inferior frontal gyrus & 3,552 & -48 & 4 & 14 & 3.15 & $<0.001$ \\
\hline \multicolumn{7}{|c|}{ The FC between the DAN and WMS } \\
\hline \multicolumn{7}{|l|}{ Positive WMS } \\
\hline R. precentral gyrus & 1,330 & 52 & 6 & 26 & 4.4 & $<0.001$ \\
\hline
\end{tabular}

FC, functional connectivity; DMN, default mode network; WMS, Wechsler Memory Scale; MNI, montreal neurological institute; R, right; L, left; DAN, dorsal attention network.

of resting-state brain networks and brain regions of patients who experienced their first-ever stroke. Increased functional connectivity was observed between the DMN and frontal cortex (left middle/inferior frontal and left precentral gyri), temporal regions (left superior temporal gyrus), bilateral cingulate gyrus, and PCC/Pcu, and decreased functional connectivity was detected between the DMN and right middle temporal gyrus, left uvula and right inferior parietal lobule in patients who had experienced their first ever stroke. Furthermore, decreased functional connectivity was identified between the DAN and prefrontal cortex (left precentral/inferior frontal and right inferior/middle frontal gyri), right inferior parietal gyrus and right insula.

Analysis of functional connectivity via resting-state fMRI is attracting more researchers to the study of brain networks as a result of its less complex task design. As a data-driven 


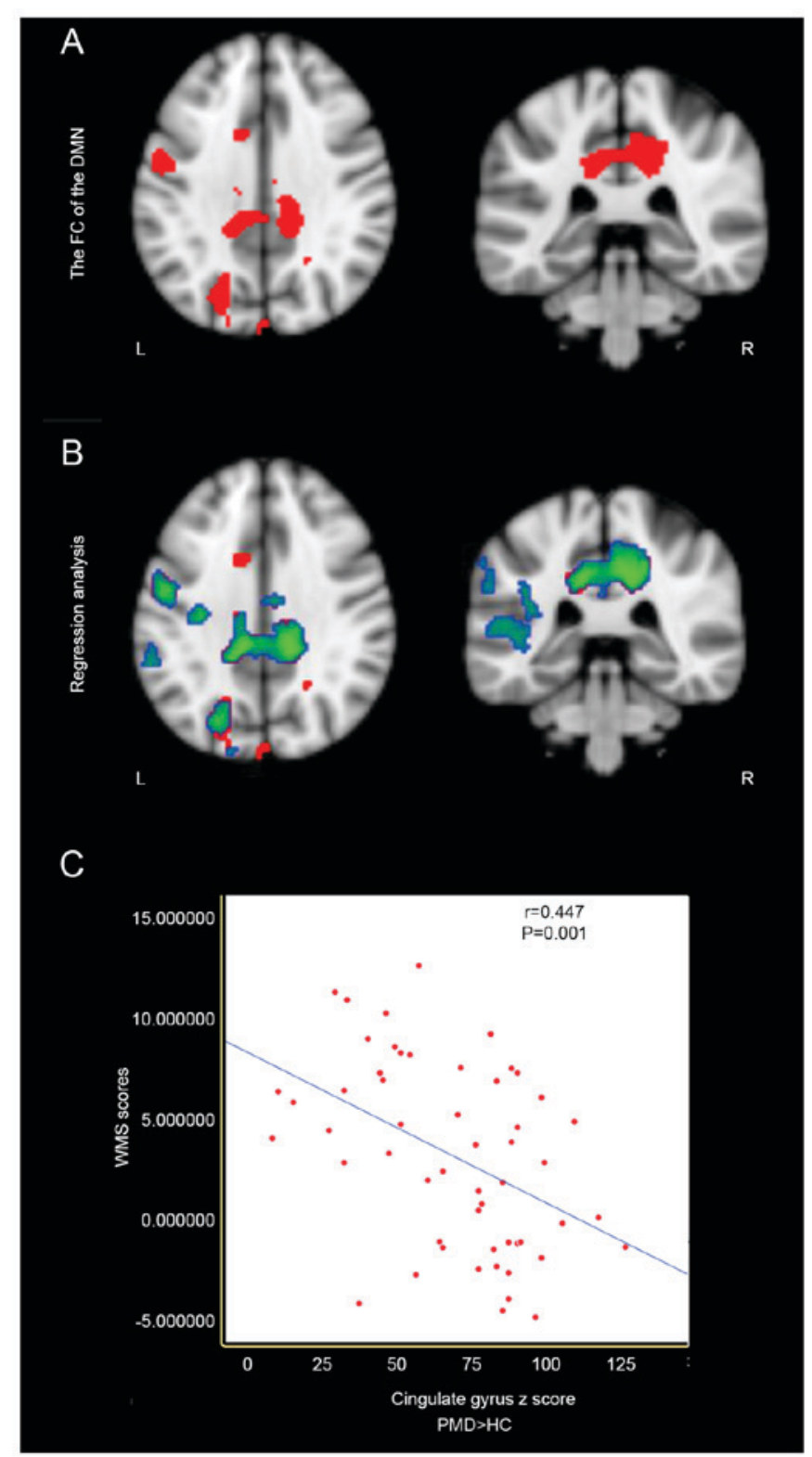

Figure 3. (A) Red color indicates brain cortices (left precentral gyrus, left middle/inferior frontal gyrus, bilateral cingulate gyrus/posterior cingulate cortices/precuneus) that exhibited increased FC with the DMN in patients with PMD compared with the $\mathrm{HC}$ group. (B) Green color indicates brain regions where DMN functional connectivity alterations were negatively associated with the corresponding WMS score changes in all participants (C) Scatter plots depict the correlation between brain region (cingulate gyrus) FC alterations and WMS score changes. FC, functional connectivity; DMN default mode network; PMD, post-stroke memory dysfunction; HC, healthy control; WMS, Wechsler Memory Scale; L, left; R, right.

approach, ICA is able to determine distinct components by capturing spatial independence and time-courses of resting-state data and thereby reliably defines different resting-state networks (30). In the present study, ICA was used on PMD and control subjects to better define DMN and DAN pathways common to both study groups (31). DMN and the DAN were chosen as they are associated with cognitive and memory function $(12,17)$. The DMN and DAN are competitive in function and distinct in spatial and sub serving cognitive processing, respectively (32). The DMN is one of the most widely researched networks using resting-state fMRI
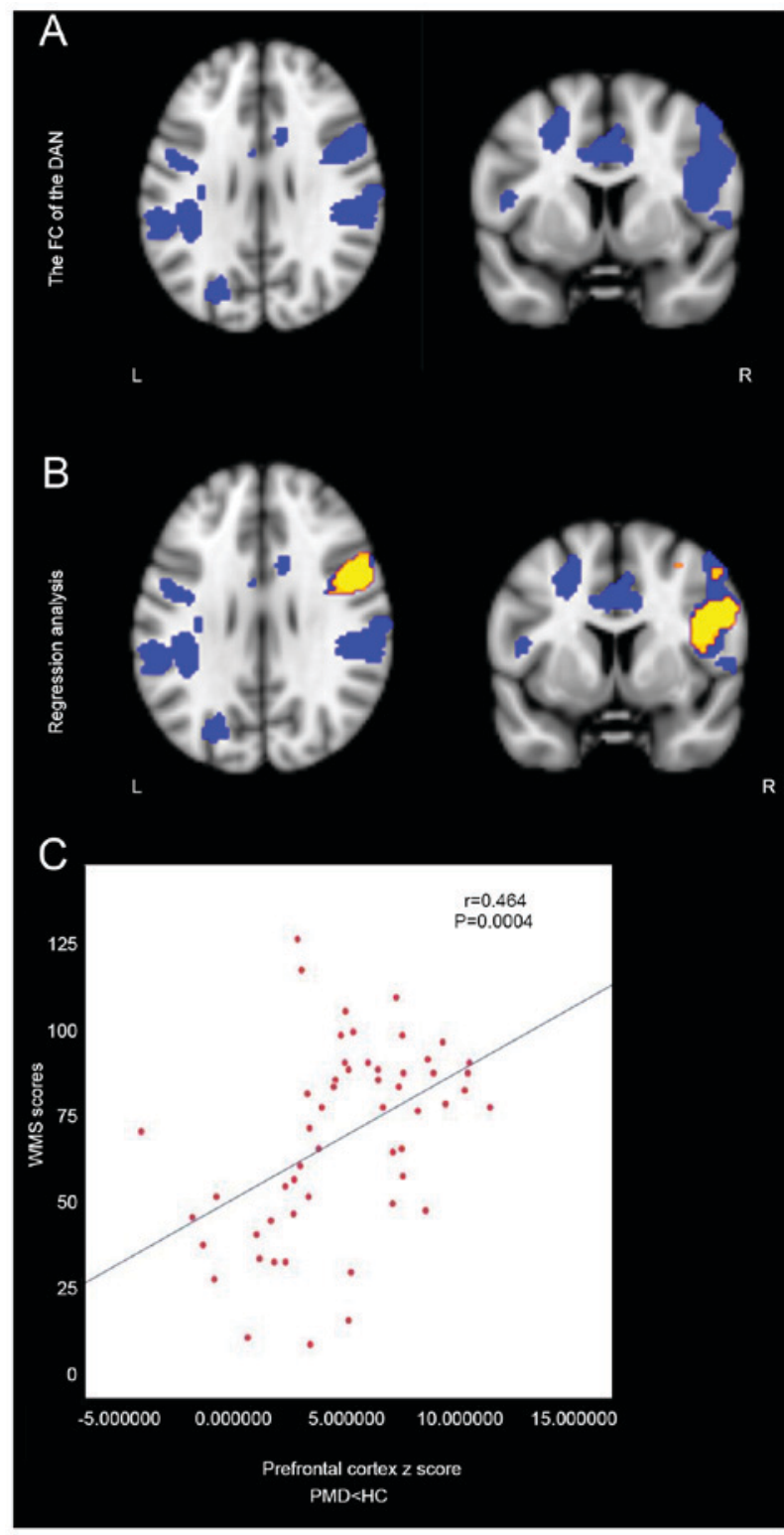

Figure 4. (A) Blue color indicates the brain cortices left precentral/inferior frontal gyrus, right inferior/middle frontal gyrus, cingulate gyrus, right inferior parietal gyrus and right insula that exhibited decreased in FC with the DAN in the PMD group compared with the HC group. (B) Yellow color indicates brain regions (right inferior/middle frontal gyrus) where DAN FC alterations were positively associated with the corresponding WMS score changes across all participants. (C) Scatter plots depict the correlation between brain region (right inferior frontal gyrus) FC alterations and WMS score changes. FC, functional connectivity; DAN, dorsal attention network; PMD, post-stroke memory dysfunction; WMS, Wechsler Memory Scale; HC, healthy control; L, left; R, right.

analysis (33). Previous studies have suggested that the DMN is associated with general cognition (34) and PMD (12); however, the sample sizes of those studies were small and their results remain controversial. Anomalous alterations in the DAN have been reported in patients with Alzheimer's disease (35), Parkinson's disease (36), epilepsy (37), and patients who have suffered a stroke. Perry et al (38) speculated that episodic memory is associated with inattention to related information. Despite considerable interest in the DAN, at present there is limited information regarding its possible changes in patients with PMD. 
In the present study, connectivity increments were identified between the DMN and bilateral PCC/Pcu. This finding is in agreement with a previous functional neuroimaging study, which reported increased functional connectivity strength in the PCC/Pcu of patients with post-stroke cognitive impairment compared with controls (11). Notably, patients with Alzheimer's disease have also demonstrated increased functional connectivity in the PCC/Pcu $(11,39)$. In the present study, it was determined that patients with PMD had increased functional connectivity in the bilateral cingulate gyrus, left claustrum, and left frontal cortex, which was correlated with poor performance in memory function tests. Similar results have been identified in studies regarding cognitive impairment and the DMN $(9,40)$. The functional connectivity analysis and the regression analysis together prove that cingulate gyrus serves an important role in memory function. These findings suggest that functional changes in the cingulate gyrus and left frontal cortex are involved in this type of deficit in patients who have suffered a stroke.

In the present study, patients with PMD exhibited reduced functional connectivity between the DMN and right middle temporal gyrus, left uvula and right inferior parietal lobule. However, there were no significant correlations between the WMS-CR and Z-scores in voxels exhibiting decreased functional connectivity in these brain regions. As well as differences in memory between the PMD and healthy groups, other confounding factors exist. For example, 25-50\% of stroke patients suffer from dysphagia (41) and a recent study (42) reported that patients with dysphagia alone had decreased functional connectivity in the DMN. As such, it cannot be assumed that the decrease in functional connectivity between the DMN and these brain regions in the present study was due solely to the effects of stroke on memory function, as the current study included 8 patients with mild to moderate dysphagia, which may have affected functional connectivity changes.

The authors of the present study were only able to identify three recently published studies that investigated DMN and post-stroke cognitive performance via resting-state fMRI. All three reported that patients who had suffered a stroke exhibited increased functional connectivity in the left frontal cortex compared with healthy controls, which is in accordance with the results of the present study $(9,11,40)$. However, using a region of interest-based analysis and ICA, Tuladhar et al (12) reported that patients who experienced their first ever right or left hemisphere stroke had decreased activity in the left frontal lobe and posterior cingulate cortex compared with controls. Their study assessed the neuropathophysiological mechanism(s) of memory impairment following stroke. Multiple factors may have contributed to the heterogeneity between the results of the present study and those of Tuladhar et al (12). For example, Tuladhar et al (12) included patients with both right and left hemisphere injury. However, the results of the present study are unable to confirm whether brain injury from the left side leads to the same alterations in functional connectivity as injury to the right (43). Furthermore, other clinical variables, including the effects of concomitant pharmacological therapies may have contributed to these contradictory results.

Previous studies have suggested that the prefrontal cortex serves a role in spatial and working memory (44), which is in accordance with the results of the present study that exhibits connectivity changes between the DAN and left precentral, inferior frontal, and right inferior/middle frontal gyri. In the present study, it was also observed that there was decreased functional connectivity between the DAN and right insula that was related to memory performance. The functional connectivity analysis and the regression analysis together indicate that the prefrontal cortex serves an important role in memory function. Christopher et al (45) reported that the fronto-insular cortex is associated with cognitive function. Notably, right frontal areas and adjacent insula associated with negative WMS-CR scores are regions within the DAN and right frontoparietal networks, which suggests that these two networks serve a role in memory processes $(27,29,46)$.

The frontal lobe is one of the most complicated brain regions and is involved in a variety of cognitive functions, including language, attention, emotion and memory $(47,48)$. Different types of memory, including working, spatial and episodic memory have different characteristics (49). Parietal fiber projections accepted by the dorsal prefrontal cortex and subsequently integrated into spatial information transmitted to the dorsal prefrontal region, ultimately forming spatial memory (50). One fMRI study reported that speech memory activates Broca's area, whereas spatial memory activates the prefrontal cortex of the right hemisphere (51). Results from studies involving spatial orientation tasks have suggested that episodic memory-encoding processes primarily depend on the prefrontal cortex, particularly in time-related information recall (47). Furthermore, Jodo et al (52) demonstrated that the brain network between the frontal lobe and hippocampus is associated with memory, particularly working memory. Despite variable findings, the present study determined that DMN activity in patients with PMD is increased in brain regions including the inferior frontal and precentral gyri, whereas DAN activity is reduced. Together, these findings confirm and complement those of previous studies $(17,18)$.

There were various limitations of the present study. The aim of the present study was to investigate the common underlying mechanism(s) of memory impairment for all of stroke patients who suffered from varying degrees of memory decline. Therefore, in the present study, 27 patients with PMD and 27 healthy control subjects were enrolled within 1 year following the onset of stroke to investigate the pathophysiology of PMD. However, different stroke courses may have some heterogeneity (53). Desmond et al (54) reported that typically, cognitive function may exhibit a long-term improvement after stroke when compared with assessments at 3 months and then annually. Another study (55) also suggested that $30 \%$ of individuals who had mild cognitive impairments between 0 and 6 months after stroke may improve, and be cognitively intact at 12-18 months. Therefore, future studies should have stricter inclusion and exclusion criterion, in particular taking into account consistent stroke course, to clarify the aforementioned issues. In conclusion, the results of the present study suggest that resting-state fMRI may help researchers to better understand the neuropathophysiological mechanisms of PMD. In particular, it has been determined that the prefrontal cortex, temporal regions, insular cortex, and PCC/Pcu serve crucial roles in memory processing. The alterations described herein suggest that stroke events affect not only the lesioned hemisphere, but also the contralesional hemisphere. 


\section{Acknowledgements}

The authors of the present study would like to thank all participants for their involvement in this study, and the nurses, neuroradiologists and technicians from the Fujian University of Traditional Chinese Medicine Subsidiary Rehabilitation Hospital for their helpful collaboration. The present study was supported by the 12th Five-year Plan supporting project of Ministry of Science and Technology of the People's Republic of China (grant number 2013BAI10B01).

\section{References}

1. WHO publishes definitive atlas on global heart disease and stroke epidemic. Indian J Med Sci 58: 405-406, 2004.

2. Gorelick PB and Nyenhuis D: Stroke and cognitive decline. JAMA 314: 29-30, 2015.

3. Kalaria RN and Ballard C: Stroke and cognition. Curr Atheroscler Rep 3: 334-339, 2001.

4. Mercier L, Audet T, Hébert R, Rochette A and Dubois MF: Impact of motor, cognitive, and perceptual disorders on ability to perform activities of daily living after stroke. Stroke 32: 2602-2608, 2001.

5. Rasquin SM, Lodder J, Ponds RW, Winkens I, Jolles J and Verhey FR: Cognitive functioning after stroke: A one-year follow-up study. Dement Geriatr Cogn Disord 18: 138-144, 2004

6. Hochstenbach J, Mulder T and Limbeek J: The neuropsychology of stroke: Changes in cognition, emotion and behaviour. TSG 75: 479-485, 1997 (In Dutch).

7. Tatemichi TK, Desmond DW, Stern Y, Paik M, Sano M and Bagiella E: Cognitive impairment after stroke: Frequency, patterns, and relationship to functional abilities. J Neurol Neurosurg Psychiatry 57: 202-207, 1994.

8. Galski T, Bruno RL, Zorowitz R and Walker J: Predicting length of stay, functional outcome, and aftercare in the rehabilitation of stroke patients. The dominant role of higher-order cognition. Stroke 24: 1794-1800, 1993.

9. Dacosta-Aguayo R, Graña M, Iturria-Medina Y, FernándezAndújar M, López-Cancio E, Cáceres C, Bargalló N, Barrios M, Clemente I, Toran P, et al: Impairment of functional integration of the default mode network correlates with cognitive outcome at three months after stroke. Hum Brain Mapp 36: 577-590, 2015.

10. Lawrence AJ, Patel B, Morris RG, MacKinnon AD, Rich PM, Barrick TR and Markus HS: Mechanisms of cognitive impairment in cerebral small vessel disease: Multimodal MRI results from the St George's cognition and neuroimaging in stroke (SCANS) study. PLoS One 8: e61014, 2013

11. Ding X, Li CY, Wang QS, Du FZ, Ke ZW, Peng F, Wang J and Chen L: Patterns in default-mode network connectivity for determining outcomes in cognitive function in acute stroke patients. Neuroscience 277: 637-646, 2014.

12. Tuladhar AM, Snaphaan L, Shumskaya E, Rijpkema M, Fernandez G, Norris DG and de Leeuw FE: Default mode network connectivity in stroke patients. PLoS One 8: e66556, 2013.

13. Dacosta-Aguayo R, Graña M, Fernández-Andujar M, López-Cancio E, Cáceres C, Bargalló N, Barrios M. Clemente I, Monserrat PT, Sas MA, et al: Structural integrity of the contralesional hemisphere predicts cognitive impairment in ischemic stroke at three months. PLoS One 9: e86119, 2014.

14. Crofts JJ, Higham DJ, Bosnell R, Jbabdi S, Matthews PM, Behrens TE and Johansen-Berg H: Network analysis detects changes in the contralesional hemisphere following stroke. Neuroimage 54: 161-169, 2011.

15. Sporns O: Network attributes for segregation and integration in the human brain. Curr Opin Neurobiol 23: 162-171, 2013.

16. Spreng RN, Sepulcre J, Turner GR, Stevens WD and Schacter DL: Intrinsic architecture underlying the relations among the default, dorsal attention, and frontoparietal control networks of the human brain. J Cogn Neurosci 25: 74-86, 2013

17. Fox MD, Snyder AZ, Vincent JL, Corbetta M, Van Essen DC and Raichle ME: The human brain is intrinsically organized into dynamic, anticorrelated functional networks. Proc Natl Acad Sci USA 102: 9673-9678, 2005.

18. Kelly AM,Uddin LQ, Biswal BB, Castellanos FX and Milham MP: Competition between functional brain networks mediates behavioral variability. Neuroimage 39: 527-537, 2008.
19. Galea M and Woodward M: Mini-mental state examination (MMSE). Aust J Physiother 51: 198, 2005.

20. Beck AT, Steer RA and Brown GK: Manual for the Beck depression inventory. TX: Psychological Corporation, San Antonio, 1996.

21. Krefetz DG, Steer RA, Gulab NA and Beck AT: Convergent validity of the beck depression inventory-II with the reynolds adolescent depression scale in psychiatric inpatients. J Pers Assess 78: 451-460, 2002

22. Katzman R, Zhang MY, Ouang-Ya-Qu, Wang ZY, Liu WT, Yu E, Wong SC, Salmon DP and Grant I: A Chinese version of the mini-mental state examination; impact of illiteracy in a shanghai dementia survey. J Clin Epidemiol 41: 971-978, 1988.

23. Gong YX JD XGDJ: Wechsler memory scale-revised manual (Chinese version). China: Hunan Medical University, 1989.

24. da Costa FA, Damasceno Bezerra IF, de Araujo Silva DL, de Oliveira R and da Rocha VM: Cognitive evolution by MMSE in poststroke patients. Int J Rehabil Res 33: 248-253, 2010.

25. Mahoney FI and Barthel DW: Functional evaluation: The barthel index. Md State Med J 14: 61-65, 1965.

26. Cordes D, Haughton VM, Arfanakis K, Carew JD, Turski PA, Moritz CH, Quigley MA and Meyerand ME: Frequencies contributing to functional connectivity in the cerebral cortex in 'resting-state' data. AJNR Am J Neuroradiol 22: 1326-1333, 2001.

27. Biswal BB, Mennes M, Zuo XN, Gohel S, Kelly C, Smith SM, Beckmann CF, Adelstein JS, Buckner RL, Colcombe S, et al: Toward discovery science of human brain function. Proc Natl Acad Sci USA 107: 4734-4739, 2010.

28. Fang J, Rong P, Hong Y, Fan Y, Liu J, Wang H, Zhang G, Chen X, Shi S, Wang L, et al: Transcutaneous vagus nerve stimulation modulates default mode network in major depressive disorder. Biol Psychiatry 79: 266-273, 2016.

29. Fox MD, Corbetta M, Snyder AZ, Vincent JL and Raichle ME: Spontaneous neuronal activity distinguishes human dorsal and ventral attention systems. Proc Natl Acad Sci USA 103: 10046-10051, 2006.

30. Beckmann CF, DeLuca M, Devlin JT and Smith SM: Investigations into resting-state connectivity using independent component analysis. Philos Trans R Soc Lond B Biol Sci 360: 1001-1013, 2005.

31. Mantini D,Perrucci MG,Del Gratta C, Romani GL and Corbetta M: Electrophysiological signatures of resting state networks in the human brain. Proc Natl Acad Sci USA 104: 13170-13175, 2007.

32. Andrews-Hanna JR: The brain's default network and its adaptive role in internal mentation. Neuroscientist 18: 251-270, 2012.

33. Buckner RL, Andrews-Hanna JR and Schacter DL: The brain's default network: Anatomy, function, and relevance to disease. Ann N Y Acad Sci 1124: 1-38, 2008.

34. Broyd SJ, Demanuele C, Debener S, Helps SK, James CJ and Sonuga-Barke EJ: Default-mode brain dysfunction in mental disorders: A systematic review. Neurosci Biobehav Rev 33: 279-296, 2009.

35. Khazaee A,Ebrahimzadeh A and Babajani-Feremi A: Application of advanced machine learning methods on resting-state fMRI network for identification of mild cognitive impairment and Alzheimer's disease. Brain Imaging Behav 10: 799-817, 2016.

36. Baggio HC, Segura B, Sala-Llonch R, Marti MJ, Valldeoriola F, Compta Y, Tolosa E and Junqué C: Cognitive impairment and resting-state network connectivity in Parkinson's disease. Hum Brain Mapp 36: 199-212, 2015.

37. Zhang Z, Lu G, Zhong Y, Tan Q, Yang Z, Liao W, Chen Z, Shi J and Liu Y: Impaired attention network in temporal lobe epilepsy: A resting FMRI study. Neurosci Lett 458: 97-101, 2009.

38. Perry RJ, Watson P and Hodges JR: The nature and staging of attention dysfunction in early (minimal and mild) Alzheimer's disease: Relationship to episodic and semantic memory impairment. Neuropsychologia 38: 252-271, 2000.

39. Chhatwal JP, Schultz AP, Johnson K, Benzinger TL, Jack C Jr, Ances BM, Sullivan CA, Salloway SP, Ringman JM, Koeppe RA, et al: Impaired default network functional connectivity in autosomal dominant Alzheimer disease. Neurology 81: 736-744, 2013.

40. Dacosta-Aguayo R, Graña M, Savio A, Fernández-Andújar M, Millán M, López-Cancio E, Cáceres C, Bargalló N, Garrido C, Barrios M, et al: Prognostic value of changes in resting-state functional connectivity patterns in cognitive recovery after stroke: A 3 T fMRI pilot study. Hum Brain Mapp 35: 3819-3831, 2014.

41. Daniels SK, Ballo LA, Mahoney MC and Foundas AL: Clinical predictors of dysphagia and aspiration risk: Outcome measures in acute stroke patients. Arch Phys Med Rehabil 81: 1030-1033, 2000. 
42. Li S, Zhou M, Yu B, Ma Z, Chen S, Gong Q, He L, Huang X, Lui S, Wang X, et al: Altered default mode and affective network connectivity in stroke patients with and without dysphagia. J Rehabil Med 46: 126-131, 2014.

43. Gialanella B, Bertolinelli $M$ and Santoro R: Rehabilitative outcome in supratentorial and infratentorial stroke: The role of motor deficits. Aging Clin Exp Res 20: 310-315, 2008.

44. Zhou LY, Wright TE and Clarkson AN: Prefrontal cortex stroke induces delayed impairment in spatial memory. Behav Brain Res 296: 373-378, 2016.

45. Christopher L, Koshimori Y, Lang AE, Criaud M and Strafella AP: Uncovering the role of the insula in non-motor symptoms of Parkinson's disease. Brain 137: 2143-2154, 2014.

46. Bor D and Seth AK: Consciousness and the prefrontal parietal network: Insights from attention, working memory, and chunking. Front Psychol 3: 63, 2012.

47. D'Argembeau A, Ruby P, Collette F, Degueldre C, Balteau E, Luxen A, Maquet P and Salmon E: Distinct regions of the medial prefrontal cortex are associated with self-referential processing and perspective taking. J Cogn Neurosci 19: 935-944, 2007.

48. Wang XL, Du MY, Chen TL, Chen ZQ, Huang XQ, Luo Y, Zhao YJ, Kumar P and Gong QY: Neural correlates during working memory processing in major depressive disorder. Prog Neuropsychopharmacol Biol Psychiatry 56: 101-108, 2015.
49. Amici S: Memory dysfunction. Front Neurol Neurosci 30: 54-56, 2012.

50. Lin ZC, Yang SL, Xue XH, Tao J and Chen LD: Central mechanism of acupuncture at baihui for memory impairment after stroke: A resting-state functional magnetic resonance imaging study. Chin J Rehabil Theory Pract: 184-188, 2015.

51. Smith EE and Jonides J: Storage and executive processes in the frontal lobes. Science 283: 1657-1661, 1999.

52. Jodo E, Suzuki Y, Katayama T, Hoshino KY, Takeuchi S, Niwa S and Kayama Y: Activation of medial prefrontal cortex by phencyclidine is mediated via a hippocampo-prefrontal pathway. Cereb Cortex 15: 663-669, 2005.

53. Rasquin SM, Welter J and van Heugten CM: Course of cognitive functioning during stroke rehabilitation. Neuropsychol Rehabil 23: 811-823, 2013.

54. Desmond DW, Moroney JT, Sano M and Stern Y: Recovery of cognitive function after stroke. Stroke 27: 1798-1803, 1996.

55. Tham W, Auchus AP, Thong M, Goh ML, Chang HM, Wong MC and Chen CP: Progression of cognitive impairment after stroke: One year results from a longitudinal study of Singaporean stroke patients. J Neurol Sci 203-204: 49-52, 2002. 